\title{
INFLUENCE OF FLOW DIRECTION CHANGE ON THE PARTICLE SEGREGATION IN HORIZONTAL PIPES
}

\author{
G. Borsuk* , B. Dobrowolski**
}

\begin{abstract}
In the pneumatic transport of solid particles, a gradual deposition of particles takes place. One of the factors affecting the intensity of particle segregations is the flow conditions upstream the pipeline. Numerical calculations allowed determining the intensity of particles deposition during transport in a horizontal pipeline with different elbow positioning at the inlet to the channel.
\end{abstract}

Keywords: pneumatic conveying, particle segregation, numerical calculations

\section{Introduction}

Horizontal pipelines are frequently occurring elements of the pneumatic transport system. During longer distance transport, a significant operational problem is the deposition of solid particles in the bottom of the pipeline (Borsuk et al., 2004). The most common side effects caused by the phenomenon of particle segregation, in addition to erosion and pulsation (Wydrych et al., 2017, Anweiler et al., 2004) may include a change in operations conditions in industrial installations and often their deterioration (Akili et al., 2001, Lain et al., 2012). The formation of particle rope is a problem, particularly with non-spherical particles (Kruggel-Emden et al., 2014). It was also compared both, experimental and numerical research of pulverized coal particles conveying in the horizontal channel (Akili et al., 2005). In the power industry, these problems can occur in the systems of dust-fired power boilers (Olcay et al., 2016). The aim of this work is a theoretical analysis of the phenomenon of gas-solid mixture flow in a horizontal pipe. In particular, the object of the research is to assess the impact of an elbow on the particle segregation process.

\section{Mathematical model}

The three-dimensional isothermal flow of the fixed gas-solid mixture is analyzed. The gas motion is described based on the conservation of mass and momentum (Borsuk et al., 2006). The basic system of equations is made up of the equation of flow continuity and the equation of momentum conservation. In this work, the system of motion is closed with the k- $\varepsilon$ turbulence model (Borsuk et al., 2016).

The equation of continuity, motion and turbulence model can be presented in a generalized conservative form, isolating convective, diffusion and source elements

$$
\frac{\partial}{\partial x}(\rho U \phi)+\frac{\partial}{\partial y}(\rho U \phi)+\frac{\partial}{\partial z}(\rho U \phi)=\frac{\partial}{\partial x}\left(\Gamma_{\phi} \frac{\partial \phi}{\partial x}\right)+\frac{\partial}{\partial y}\left(\Gamma_{\phi} \frac{\partial \phi}{\partial y}\right)+\frac{\partial}{\partial z}\left(\Gamma_{\phi} \frac{\partial \phi}{\partial z}\right)+S_{\phi}+S_{\phi p}
$$

where $\phi$ is a generalized dependent variable, $\Gamma_{\phi}$ the diffusion transport coefficient, and the source element

Grzegorz Borsuk, PhD.: Opole University of Technology, Faculty of Mechanical Engineering, Department of Thermal Engineering and Industrial Facilities, ul. Mikołajczyka 5, 45-271 Opole, g.borsuk@po.opole.pl

** Boleslaw Dobrowolski, Prof.: Opole University of Technology, Faculty of Mechanical Engineering, Department of Thermal Engineering and Industrial Facilities, ul. Mikołajczyka 5, 45-271 Opole, b.dobrowolski@po.opole.pl 
$\mathrm{S}_{\phi}$ captures all other - apart from convective and diffusion - components of differential equations. The coefficients $\Gamma_{\phi}$ and $\mathrm{S}_{\phi}$ depend on the variable $\phi$ and determined for each of the equations according to table 1.

Tab. 1: Statement of equation's (1) coefficients.

\begin{tabular}{lccccc}
\hline Equation & $\phi$ & $\Gamma_{\phi}$ & $\mathrm{S}_{\phi}$ & $\mathrm{S}_{\phi \mathrm{p}}$ \\
\hline Continuity & 1 & 0 & 0 & 0 \\
\hline $\begin{array}{l}\text { Momentum in the } \\
\text { direction of axis } \mathrm{x}_{\mathrm{i}}\end{array}$ & $\mathrm{u}_{\mathrm{i}}$ & $\mu_{\mathrm{ef}}$ & $F_{i}-\frac{\partial \bar{p}}{\partial x_{i}}+\frac{\partial}{\partial x_{j}}\left(\mu_{e f} \frac{\partial \bar{u}_{j}}{\partial x_{i}}\right)$ & $\overline{S_{u_{i}, p}}$ \\
$\begin{array}{llcc}\text { Kinetic enegery of } \\
\text { turbulence }\end{array}$ & $\mathrm{k}$ & $\frac{\mu_{e f}}{\sigma_{k}}$ & $\frac{\partial \bar{u}_{i}}{\partial x_{j}} \mu_{t}\left(\frac{\partial \bar{u}_{i}}{\partial x_{j}}+\frac{\partial \bar{u}_{j}}{\partial x_{i}}\right)-\rho \varepsilon$ & 0 \\
\hline $\begin{array}{l}\text { Dissipation of the } \\
\text { kinetic energy of } \\
\text { turbulence }\end{array}$ & $\varepsilon$ & $\frac{\mu_{e f}}{\sigma_{\varepsilon}}$ & $\frac{\varepsilon}{k}\left(C_{1} \frac{\partial \bar{u}_{i}}{\partial x_{j}} \mu_{t}\left(\frac{\partial \bar{u}_{i}}{\partial x_{j}}+\frac{\partial \bar{u}_{j}}{\partial x_{i}}\right)-C_{2} \rho \varepsilon\right)$ & 0 \\
\end{tabular}

The particle motion is described by the Lagrange method. The equation of motion with considering the inertia force, aerodynamic drag force and gravity takes the form of (2)

$$
\frac{d u_{p}}{d t}=\frac{1}{\tau_{p}}\left(u-u_{p}\right)+g
$$

where $\tau_{\mathrm{p}}$ is the time of dynamic relaxation.

Interphase interactions were included in the form of additional source components $S_{\phi p}$ of the continuous phase equations. Fig. 1 presents the flow systems analyzed in the calculations. Both systems contain a straight horizontal pipe with the length of 60 diameters and an elbow located upstream to this pipe.

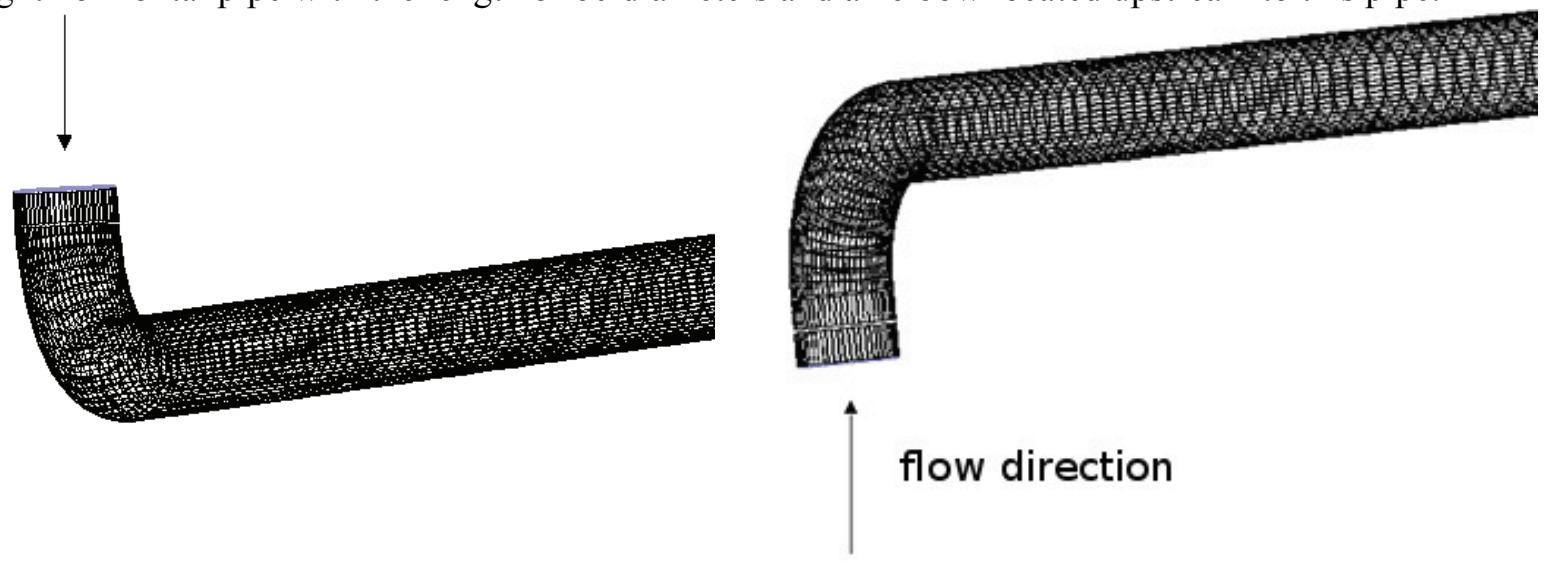

Fig. 1: Flow systems considered in calculations

\section{Results of the research and their analysis}

In order to analyze the phenomenon of particle segregation during pneumatic conveying in the gas stream, a series of numerical calculations were carried out using the ANSYS-Fluent program (Ansys, 2017). Spherical particles with the density $1550 \mathrm{~kg} / \mathrm{m}^{3}$ were used. Fig. 2 presents particle concentration distribution for diameter $\mathrm{d}=90 \mu \mathrm{m}$ in selected cross-sections of a flow system. Fig. 2a shows particle concentration distribution in a horizontal pipe, Fig. $2 \mathrm{~b}$ a system with a vertical elbow and downflow, while Fig. 2c - a system with a vertical elbow and upflow.

In the case of the flow of particles through the horizontal pipe, it is seen that most of the particles move to the bottom of the pipe at the moment of moving away from the inlet. During conveying through the 
vertical elbow with downflow the areas of maximum concentration move to the bottom part of the horizontal channel while during upflow the areas of maximum concentration can be observed initially in the upper section of the channel and then, as the distance from the inlet increases, particles deposit gradually in the lower part of the channel.

05D

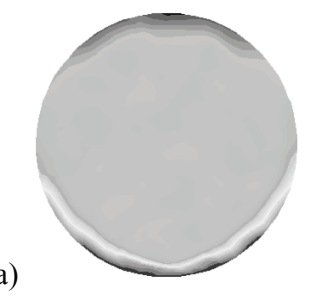

b)

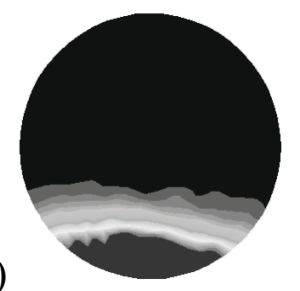

c)

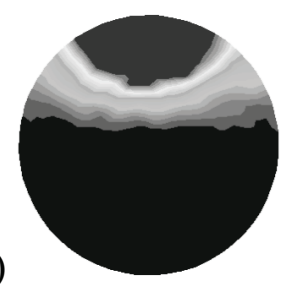

$15 \mathrm{D}$
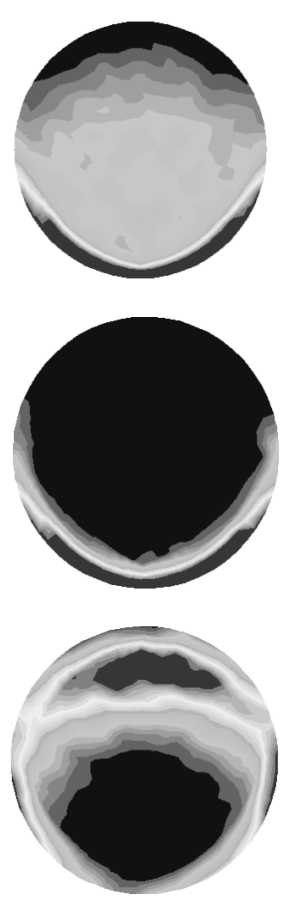

0
$25 \mathrm{D}$
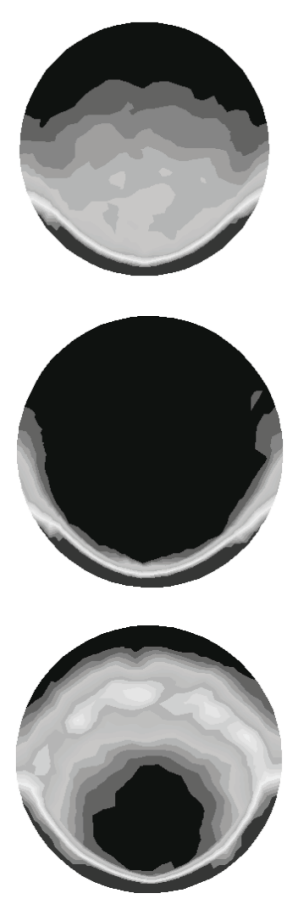

$1 \mathrm{~kg} / \mathrm{m}^{3}$
$35 \mathrm{D}$
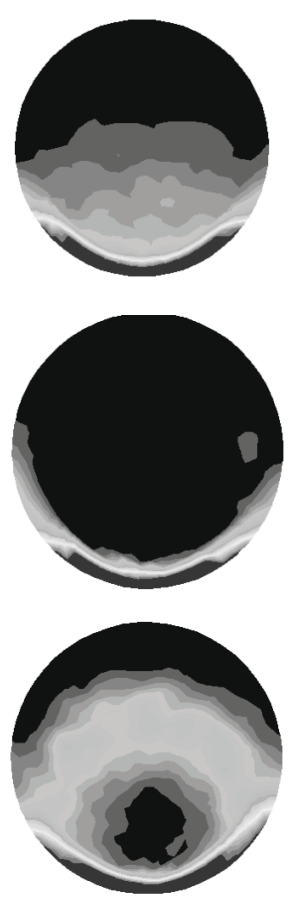

Fig. 2: Particle concentration distribution for diameter $90 \mu \mathrm{m}$ in selected sections flow configuration: a) horizontal pipe,

b) system with vertical elbow and downflow, c) system with vertical elbow and upflow

In addition to the qualitative determination of segregation level, an additional procedure was used to determine two values: the maximum $\mathrm{Y}_{\max }$ and the mean value $\mathrm{Y}_{\mathrm{av}}$ of the particle concentration in the cross-section of the channel.

$$
\phi=\frac{Y_{\max }}{Y_{a v}}
$$

The $\phi$ value shows us the degree of unevenness in the distribution of the particle concentrations in particular cross-sections. Fig. 3 shows the changes in the coefficient of inhomogeneity of the concentration distribution along the horizontal pipeline during conveying for $40 \mu \mathrm{m}$ particles, while Fig. 4 shows the values of the relevant data for $90 \mu \mathrm{m}$ particles.

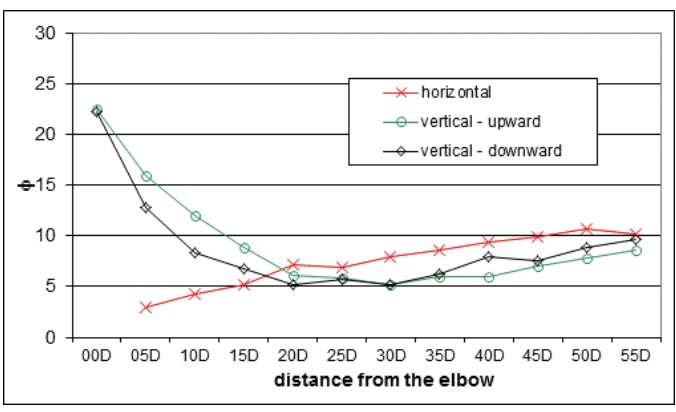

Fig. 3: . Concentration inhomogeneity coefficient in selected cross-sections horizontal pipe, $d=40 \mu m$ 


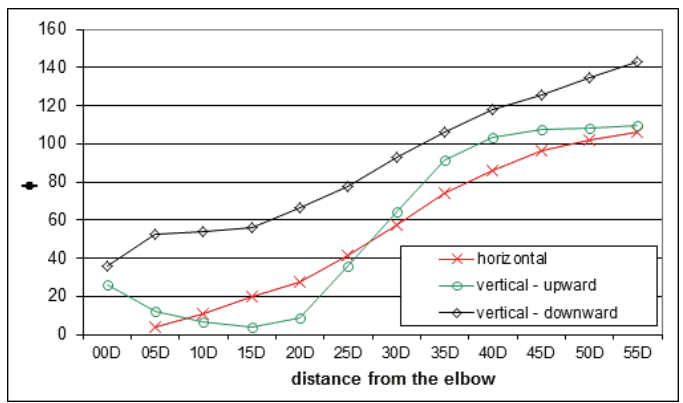

Fig. 4: Concentration inhomogeneity coefficientin selected cross-sections horizontal pipe, $d=90 \mu m$

Fig. 3 and Fig. 4 show a large increase of the concentration inhomogeneity coefficient in the initial section of the pipeline, which is the effect of the centrifugal force when changing the flow direction. For particles with a diameter $40 \mu \mathrm{m}$ (Fig. 3) the $\phi$ value decreases at a distance larger than 20 diameters from the inlet. The value of the coefficient $\phi$ practically does not depend on the direction of the inflow. The calculated $\phi$ values practically do not differ from the values corresponding to the flow in the straight horizontal pipe.

For particles with a diameter $90 \mu \mathrm{m}$ (Fig. 4) the effect of centrifugal force is more visible. The $\phi$ values in the initial section of the flow system are greater. The influence of gravity on the gradual particle deposition along the pipeline, in this case, is more important.

\section{Conclusions}

Numerical research carried out allows formulating the following conclusions. Change of the flow direction causes a significant increase of the particle concentration in the external side of the elbow. The effect of gradual particle segregation and its sedimentation at the bottom side of the pipeline depends on the particle size distribution. In the case of a system with a vertical elbow, the phenomenon of segregations depends on the flow direction at the inlet.

\section{References}

Akili H., Levy E.K., Sahin B. (2001) Gas-solid flow behavior in a horizontal pipe after a $90^{\circ}$ vertical-to-horizontal elbow. Powder Technology, 116, 43-52

Akili H., Levy E.K., Sahin B. (2005) Investigation of gas-solid flow structure after a $90^{\circ}$ vertical-to-horizontal elbow for low conveying gas velocities. Advanced Powder Technology, Vol. 16, No. 3, pp. 261-274

ANSYS Fluent, Ansys Inc., (2017)

Anweiler, S. and Ulbrich, R. (2004). Flow pattern for different fluidization apparatuses. Inzynieria Chemiczna $i$ Procesowa, 25, 3, pp. 577-582.

Borsuk, G., Dobrowolski, B., Wydrych, J. (2006) Gas-solid mixture flow through a two-bend system, Inzynieria Chemiczna i Procesowa, vol. 27, Issue 3, pp. 645-656

Borsuk, G., Olszowski, T. (2004) Experimental and numerical examination of the influence mass-flow rate on concentration distribution in horizontal channel, Inzynieria Chemiczna i Procesowa, vol. 25, Issue 4, pp. 20892096

Borsuk, G., Pochwala, S., Wydrych, J. (2016) Numerical methods in processes of design and operation in pneumatic conveying systems, In: Engineering Mechanics 2016, IT AS CR, Prague, pp. 78-81

Kruggel-Emden, H., Oschmann, T. (2014) Numerical study of rope formation and dispersion of non-spherical particles during pneumatic conveying in a pipe bend. Powder Technology, Vol. 268, pp. 219-236

Laín S., Sommerfeld M. (2012). Numerical calculation of pneumatic conveying in horizontal channels and pipes: Detailed analysis of conveying behaviour. International Journal of Multiphase Flow, 39, 105-120

Olcay, A., Kahraman, M., Atas, S. (2016) Numerical and experimental investigation of a pulverized coal mill duct system in the Soma B thermal power plant by plant performance tests, ISI Bilimi ve Teknigi Dergisi - Journal of Thermal Science and Technology, vol. 36, Issue 2, pp. 135-146

Wydrych, J., Borsuk, G., Pochwala, S. (2017) Analysis of the erosive wear modeling results of the pneumatic conveying systems, In: Engineering Mechanics 2017, Brno Technical University, Brno, pp. 1074-1077 